\title{
Biological influence of brain-derived neurotrophic factor on breast cancer cells
}

\author{
XIAOMEI YANG ${ }^{1,2}$, TRACEY A. MARTIN ${ }^{1}$ and WEN G. JIANG ${ }^{1}$ \\ ${ }^{1}$ Metastasis and Angiogenesis Research Group, Institute of Cancer and Genetics, Cardiff University \\ School of Medicine, Cardiff, CF14 4XN, UK; ${ }^{2}$ Department of Biochemistry and \\ Molecular Biology, Capital Medical University, Beijing 100069, P.R. China
}

Received April 19, 2012; Accepted June 15, 2012

DOI: $10.3892 /$ ijo.2012.1581

\begin{abstract}
Brain-derived neurotrophic factor (BDNF) is a member of the neurotrophin superfamily which has been indicated in the pathophysiology of the nervous system and is important in a number of neurological and psychological conditions. Recently, BDNF was also shown to play a role in the development and progression of solid tumour myeloma. It has been reported that BDNF is aberrantly expressed in human breast cancer and that a raised level of BDNF is associated with poor clinical outcome and reduced survival. The present study investigated the role of BDNF in human breast cancer. A panel of human breast cancer cells was used and the expression profile of BDNF was evaluated using RT-PCR. We constructed a set of anti-BDNF transgenes which were used to transfect breast cancer cells in order to generate BDNF knocked down cells. The impact of BDNF knockdown on growth and apoptosis was evaluated. Statistical analysis was performed using SPSS. $\mathrm{P}<0.05$ was considered statistically significant. BDNF gene transcripts were successfully detected in the breast cancer cell lines MCF-7, MDA-MB-231 and ZR75-1 MDA-MB-231 and MCF-7 wild-type cells were subject to transfection of anti-BDNF transgenes, followed by the establishment of BDNF knocked down sublines. Knockdown of BDNF in MDA-MB-231 and MCF-7 cell lines resulted in decreased rates of growth and proliferation.
\end{abstract}

Correspondence to: Dr Tracey A. Martin, Metastasis and Angiogenesis Research Group, Institute of Cancer and Genetics, Cardiff University School of Medicine, Cardiff, CF14 4XN, UK

E-mail: martinta1@cf.ac.uk

Abbreviations: BDNF, brain derived neurotrophic factor; NGF, nerve growth factor; QPCR, quantitative polymerase chain reaction; $\mathrm{NF \kappa B}$, nuclear factor $\kappa \mathrm{B}$; TrkB, tyrosine kinase or BDNF/NT-3 growth factors receptor; PI3K, phosphatidylinositol 3-kinase; AKT, protein kinase $\mathrm{B}$ (PKB); MAPK, mitogen-activated protein (MAP) kinases; ERK, extracellular signal-regulated kinase; ECACC, European Collection of Cell Cultures; DMEM, Dulbecco's modified Eagle's medium; FITC, fluorescein isothiocyanate

Key words: brain derived neurotrophic factor, breast cancer, proliferation, apoptosis
Analysis of apoptosis showed that cell apoptosis was increased in cells stably transfected with ribozymes for BDNF compared with the vector control. It is concluded that BDNF, a neurotrophic growth factor aberrantly expressed in cancers such as breast cancer, has a profound impact on the cellular behaviour of breast cancer cells and that BDNF is associated with a reduction of the apoptosis of breast cancer. BDNF is, therefore, a potential therapeutic target in breast cancer and its effect in human breast cancer requires further investigation.

\section{Introduction}

The incidence of breast cancer continues to rise. Breast cancer is one of the common carcinoma in women. The treatment of breast cancer includes routine surgery, radiotherapy, biotherapy and hormone therapy. These treatments have shortcomings due to side effects and lack of response due to the different genetic makeup of the individual and their disposition to breast cancer. Recently, there has been a move towards an individual treatment regime. The search therefore continues to find markers and/ or targets for therapy $(1,2)$. Brain-derived neurotrophic factor, BDNF is a member of the neurotrophins superfamily composed of 247 amino acids which has separated and purified from pig brain (3). BDNF activates cellular biological effects mainly through a cell surface tyrosine kinase receptor, tropo-myosinrelated kinase B (TrkB). It has been reported that brain-derived neurotrophic factor (BDNF) is aberrantly expressed in human breast cancer and that a raised level of BDNF is associated with poor clinical outcome and reduced survival (4,5). BDNF is secreted by target non-neuronal cells of neuron and has been implicated in the pathophysiology of the nervous system and is important in a number of neurological and psychological conditions $(6,7)$.

Recently, in addition to BDNF involvement in the nerve system carcinomas, it has also been shown to play a role in the proliferation, invasion and metastasis of non-neuronal solid tumours such as breast cancer, myeloma, melanoma, lung cancer, ovarian cancer, hepatocellular and prostate cancer (5,8-11). The expression level of BDNF is also of high concern in cancer research (12).

Nerve growth factor (NGF) and BDNF have similar primary structures and relative functions which stimulate neuron cell survival, differentiation and neuroplasticity (6). NGF has been 
found incorporated to promote survival and proliferation of breast cancer cells which has been used in hormone therapy of breast cancer (5). The impact of expression level of BDNF on breast cancers especially diminishing the expression level would be helpful to the treatment of the breast cancer.

The present study aimed to investigate the biological role of BDNF expression in human breast cancer cells. We found high expression of BDNF in MDA-MB-231, MCF-7 and ZR75-1 breast cancer cells and so anti-BDNF ribozymes were constructed to knock down the expression of BDNF in MDA-MB-231 and MCF-7 cells. The expression profile of BDNF was evaluated and screened, then the biological influence was studied in breast cancer cells.

\section{Materials and methods}

Cell lines and culture. Human breast cell lines, MDA-MB231, MCF-7 and ZR75-1 were obtained from the European Collection of Animal Cell Cultures (ECACC, Salisbury, UK). Cells were maintained in Dulbecco's modified Eagle's medium (DMEM) containing $10 \%$ fetal calf serum, $100 \mathrm{U} / \mathrm{ml}$ penicillin and $100 \mu \mathrm{g} / \mathrm{ml}$ streptomycin (Gibco BRC, Paisley, UK) at $37^{\circ} \mathrm{C}$ and $5 \% \mathrm{CO}_{2}$.

RNA preparation and reverse transcription PCR (RT-PCR). Total cellular RNA was extacted from the cultured cells using Total RNA Isolation reagent (ABgene, Epsom, UK). The concentration of RNA was determined through an ultraviolet spectrophotometer (WPA UV 1101, Biotech Photometer, Cambridge, UK). cDNA was obtained from RT-PCR using a transcription kit (Sigma, Poole, UK). The quality of DNA was verified using GAPDH primers (forward primer: 5'-AGC TTG TCA TCA ATG GAA AT-3'; reverse primer: 5'-CTT CAC CAC CTT CTT GAT GT-3'). The mRNA level of BDNF were assessed using the BDNF primers (forward primer: 5'-TTC ATA CTT TGG TTG CAT GA-3'; reverse primer: 5'-TTC AGT TGG CCT TTT GAT AC-3'). PCR were running in a GeneAmp PCR System 2400 thermocycler (Perkin-Elmer). The PCR products were separated by $1 \%$ agarose gel and stained with ethidium bromide then photographed by a digital camera mounted over a UV transilluminator.

Real-time quantitative polymerase chain reaction. The mRNA level of gene expression was determined by the real-time quantitative polymerase chain reaction (QPCR) method using the prepared cDNA as the template. An additional primer sequence was added to every QPCR reaction system, known as the $\mathrm{Z}$ sequence (5'-ACT GAA CCT GAC CGT ACA-3') which is complementary to the universal $\mathrm{Z}$ probe (Intergen Inc., Oxford, UK). The reaction was carried out on IcyclerIQ ${ }^{\mathrm{TM}}$ (Bio-Rad, Hemel Hemstead, UK) in real-time detection of the 96-well plate. GAPDH expression was used as an internal control. The reaction condition was: $94^{\circ} \mathrm{C}$ for $7 \mathrm{~min}, 80$ cycles of $94^{\circ} \mathrm{C}$ for $15 \mathrm{sec}, 55^{\circ} \mathrm{C}$ for $35 \mathrm{sec}$ (the data capture step) and $72^{\circ} \mathrm{C}$ for $20 \mathrm{sec}$. The levels of the transcripts were generated from an internal standard that was simultaneously amplified with the samples.

Knockdown of BDNF expression using ribozyme and screen of stable transfected cell lines. Targeting human BDNF hammer- head ribozymes were designed based on the secondary structure of the gene generated using the Zuker RNA mFold program. The ribozymes were accordingly synthesized and then cloned into pEF6/V5-his-Topo T/A vector (Invitrogen, Paisley, UK). Then transfected to MDA-MB-231 and MCF-7 cells respectively using an Easyjet Plus electroporator (EquiBio, Kent, UK). After selection with culture medium containing $5 \mu \mathrm{g} / \mathrm{ml}$ blasticidin, the verified transfectants were cultured in medium containing $0.5 \mu \mathrm{g} / \mathrm{ml}$ blasticidin. Primer sequences of the anti-BDNF ribozymes were: forward primer: 5'-CTG CAG TTG GCC TTT TGA TAC AGG GAC CTT TTC AAG GAC TGT CTG ATG AGT CCG TGA GGA-3'; reverse primer: 5'-CTG CAG TTG GCC TTT TGA TAC AGG GAC CTT TTC AAG GAC TGT CTG ATG AGT CCG TGA GGA-3'.

Western blotting experiment. Human breast cancer cells were collected and lysed in lysate buffer. The protein concentration was quantified using DC Protein Assay kit (Bio-Rad) and an ELx800 spectrophotometer (Bio-Tek). Lysates were detected by SDS-PAGE and western blot analysis. The transferred membranes were incubated with the primary antiby anti-BDNF and anti-TrkB antibody (Santa Cruz Biotechnologies, Santa Cruz, CA, USA). GAPDH expression was used as an internal control (Santa Cruz Biotechnologies). Then incubated with the peroxidase-conjugated secondary antibody (Sigma-Aldrich). Western blotting results were detected by chemiluminescence and visualized using a Supersignal West Dura system (Pierce Biotechnology, Inc.) and photographed using a UVI-Tech imager (Uvitech, Cambridge, UK). Protein level were quantified and analyzed with NIH Image 1.62 .

Cell growth assay. Breast cancer cell growth rates were assessed using an in vitro growth assay. Cells were planted in sextuplicate into 96-well plates at a density of 2,000 cells per well. Plates were then incubated for 24, 48, 72 and $120 \mathrm{~h}$ before being fixed in $4 \%$ formaldehyde (v/v) and stained with $0.5 \%$ (w/v) crystal violet. The crystal violet stain was then dissolved using $10 \%$ acetic acid (v/v) and cell density was determined by measuring the absorbance of this solution at $540 \mathrm{~nm}$ using a Bio-Tek ELx800 multi-plate reader (Bio-Tek Instruments Inc., Winooski, VT).

Flow cytometric analysis of apoptosis. All cells including those floating in the culture medium were harvested after incubation. Cells were washed in cold BSS and resuspended in 1X annexin V-binding buffer at a density of $1 \times 10^{6}$ cells/ml after centrifugation. FITC annexin V $(5 \mu \mathrm{l})$ and $1 \mu \mathrm{l}$ of the PI working solution (100 $\mu \mathrm{g} / \mathrm{ml})$ (Molecular Probes, OR, USA) were added to $100 \mu \mathrm{l}$ the cell suspension. After a 15-min incubation at room temperature, $400 \mu \mathrm{l}$ of $1 \mathrm{X}$ annexin $\mathrm{V}$-binding buffer was added, mixed gently and the samples were immediately placed on ice. The stained cells were analyzed using the flow cytometer and FlowMax software.

Statistical analysis. Statistical analysis was performed using SPSS. $\mathrm{P}<0.05$ was considered statistically significant.

\section{Results}

mRNA expression of BDNF in human breast cancer cell lines. Human cancer cell lines MDA-MB-231, MCF-7 and ZR-751 


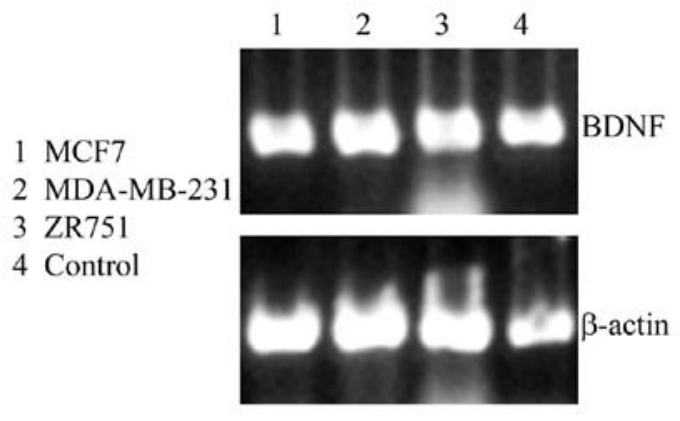

Figure 1. BDNF is expressed in human breast cancer cell lines. Detection of BDNF mRNA transcript in MCF-7, MDA-MB-231 and ZR751 breast cancer cell lines using RT-PCR. GAPDH was used as housekeeping control.

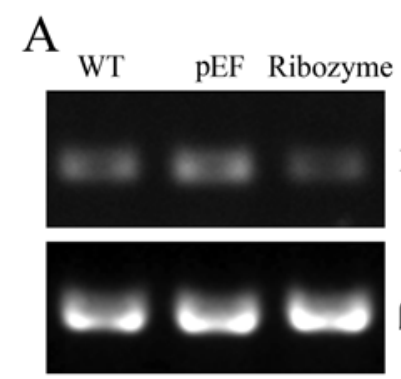

MDA-MB-231

\section{B}

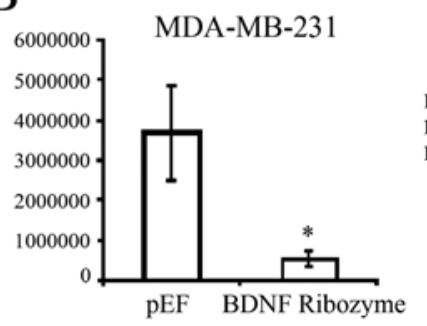

C

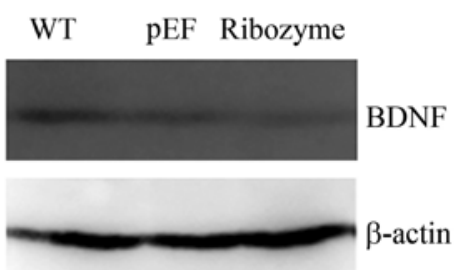

Figure 2. Knock down of BDNF expression in MDA-MB-231 and MCF-7 cell lines. (A) RT-PCR showed BDNF knockdown at the mRNA level in MDAMB-231 and MCF-7 cell lines. (B) QPCR showed BDNF knockdown at the mRNA level in MDA-MB-231 and MCF-7 cell lines. (C) Western blotting showed BDNF knockdown at the mRNA level in MDA-MB-231 cell lines.

were examined for the presence of BDNF using RT-PCR (Fig. 1). BDNF was strongly expressed in all three cell lines. Fetal kidney tissue was used as a positive control. The negative control had no DNA template (data not shown).

BDNF knockdown and establishment of stable cell lines. The ribozymes targeting BDNF were cloned into pEF6/V5-his-Topo T/A vector. MDA-MB-231 and MCF-7 wild-type cells were subjected to transfection using plasmids containing ribozymes

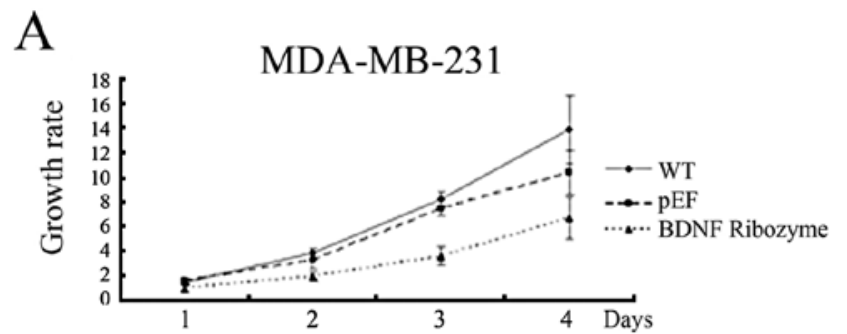

B
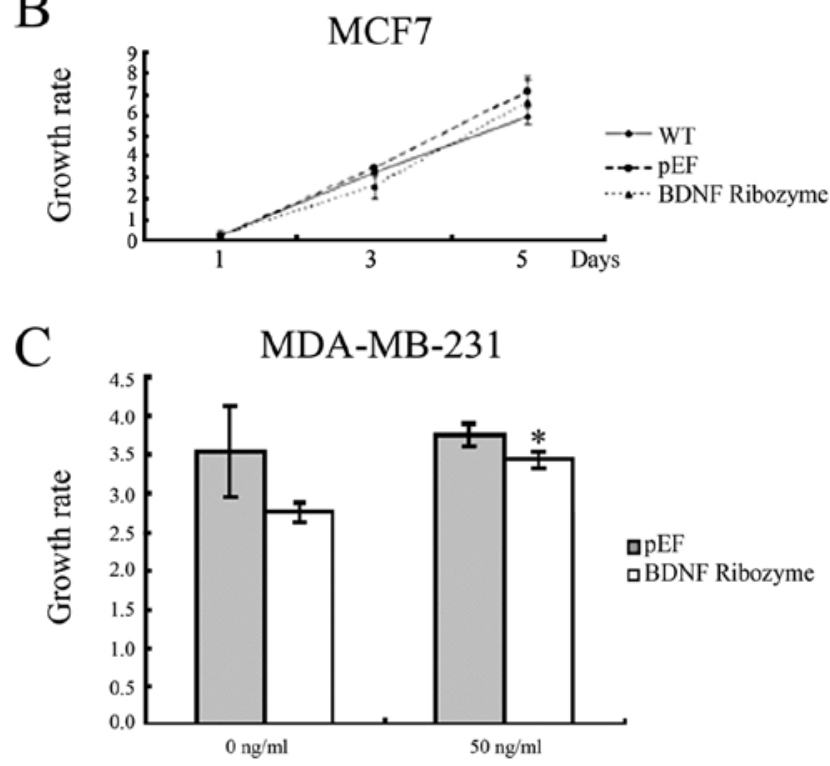

Figure 3. Effects of BDNF knockdown on cell growth. (A) Knockdown BDNF inhibited MDA-MB-231 cell growth compared with the vector control. (B) Knockdown BDNF inhibited MDA-MB-231 cell growth compared with the vector control. (C) Knockdown BDNF inhibited MDA-MB-231 cell growth was abolished by culture medium containing BDNF $(50 \mathrm{ng} / \mathrm{ml})$ compared with the vector control.

targeting BDNF or an empty vector control, respectively, followed by the establishment of BDNF knockdown sub-lines and empty vector ( $\mathrm{pEF}$ ) control cells. The expression of BDNF at the mRNA level was reduced in both BDNF knocked down MDA-MB-231 and MCF-7 cells using RT-PCR and QPCR. (Fig. 2A and B). The protein levels were also decreased in BDNF knocked down MDA-MB-231 cells using western blotting (Fig. 2C). We then characterized the effect of BDNF knockdown in these cells through a series of in vitro studies.

Effects of BDNF knockdown on the growth of human breast cancer cells. In the in vitro growth assay, knockdown of BDNF in MDA-MB-231 cells resulted in a reduction of cell growth rate (growth rate in BDNF knocked down MDA-MB-231 cells by day 3 was $3.55 \pm 0.69$, compared with $7.47 \pm 0.65$ in $\mathrm{pEF}$, $\mathrm{p}<0.001)$. Loss of BDNF in MCF-7 cells resulted in reduction of cell growth rate (growth rate in BDNF knocked-down $\mathrm{MCF}-7$ cells by day 3 was $2.59 \pm 0.58$, compared with $3.45 \pm 0.07$ in $\mathrm{pEF}$, $\mathrm{p}=0.012$ ) (Fig. 3A). These data demonstrate that BDNF may increase breast cancer cell growth.

To confirm this effect, a rescue assay was performed with the addition of BDNF protein to the cell culture medium (50 ng/ ml) (Abcam, Cambridge, UK). As a result, the reduced growth rate in the knockdown cells was attenuated (Fig. 3B). These data indicated that BDNF is involved in increased cell growth. 

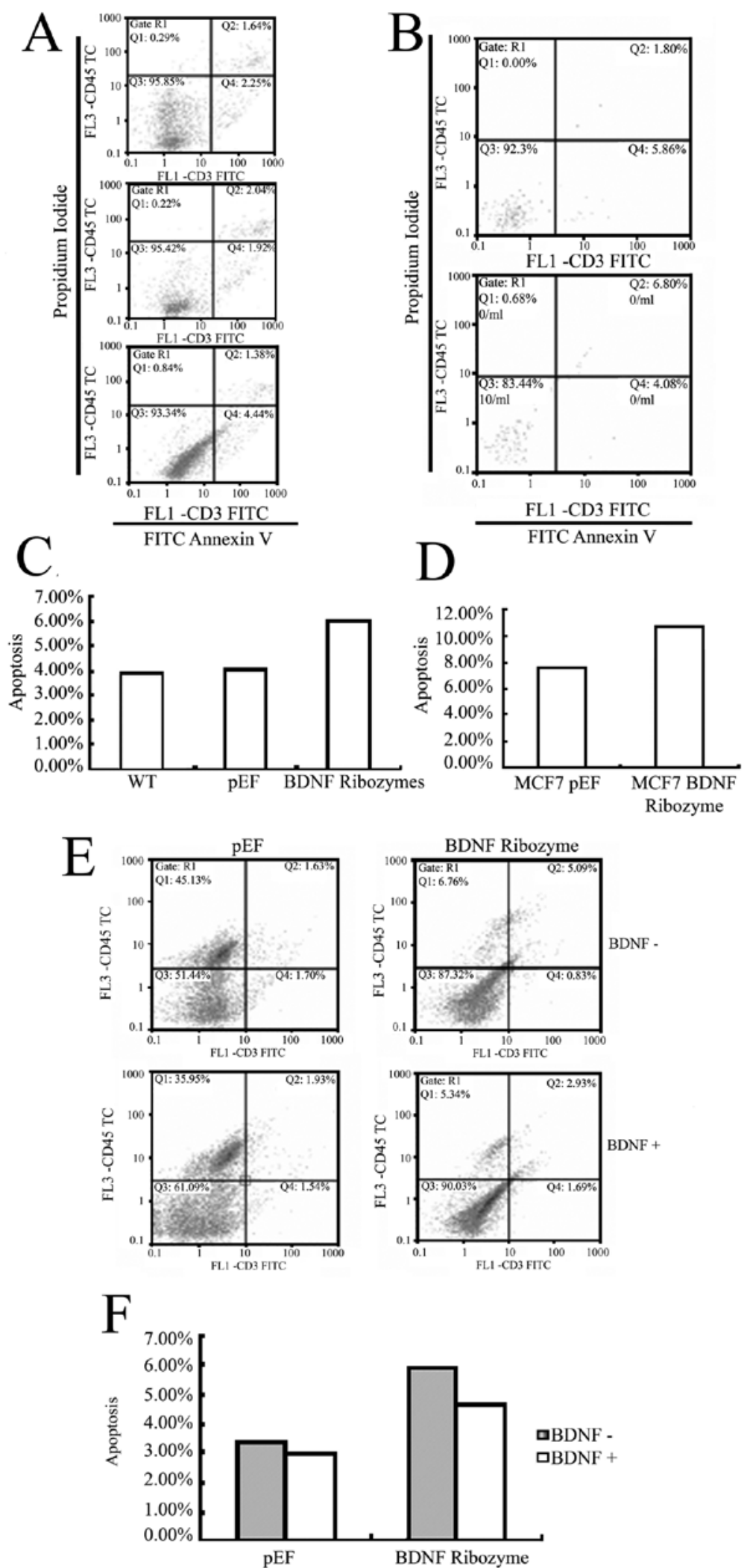

Figure 4. Effects of BDNF knockdown on cell apoptosis. (A) BDNF knockdown induced apoptosis in MDA-MB-231 cell lines. (B) BDNF knockdown induced apoptosis in MCF-7 cell lines. (C and D) Statistics for both (A) and (B). (E) The apoptotic population in MDA-MB-231 cell lines were analyzed using flow cytometry after exposure to BDNF (50 ng/ml) for $48 \mathrm{~h}$. Apoptosis was reduced compared with vector control. (F) Statistics.

Effects of BDNF knocked-down on cell apoptosis. To investigate whether apoptosis is involved in the effect of BDNF knocked down in MDA-MB-231 and MCF-7 cells, we determined the proportion of apoptotic cells. As shown in Fig. 4A-D, there was an increase in cell population towards apoptosis in the BDNF knocked down MDA-MB-231 cells, which was $6.01 \%$ in the BDNF knocked down cells, compared with $4.05 \%$ in the pEF control. There was also an increase of apoptosis in the BDNF 

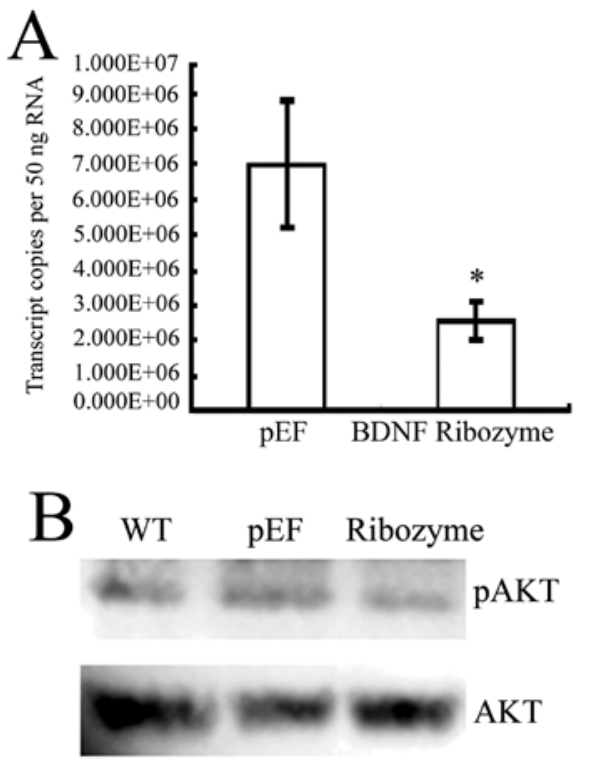

knocked down MCF-7 cells, which was $10.88 \%$ in the BDNF knocked down cells, compared with $7.66 \%$ in the pEF control. This result suggests that BDNF decreases apoptosis in these cells.

Moreover, we confirmed whether or not this effect was specific to BDNF knockdown by rescue experiments. BDNF protein was added in the cell culture medium $(50 \mathrm{ng} / \mathrm{ml})$ and resulted in negative effect of BDNF knockdown compared to the pEF controls (Fig. 4E and F).

Effects of BDNF knockdown on cellular signal pathways. We screened the cells at the mRNA transcript level for p53 and NF $\mathrm{B}$ in BDNF knocked down MDA-MB-231 cells using QPCR. The results showed that the level of p53 was decreased in BDNF knock down MDA-MB-231 and MCF-7 cells, indicating that BDNF stimulates the message for p53. mRNA levels of the NF $\kappa$ B was increased in BDNF knock down MDA-MB-231 and MCF-7 cells, indicating that BDNF affects the level of NFKB. From these results, we can infer that BDNF increases apoptosis through decreasing the anti-apoptosis factor $\mathrm{NF} \kappa \mathrm{B}$ and increase p53 (Fig. 5A and C).

Further, we examined the serine phosphorylation status of AKT in MDA-MB-231 knocked down cells. We found that the phosphorylation level of AKT was weak in BDNF knockeddown cells (Fig. 5B).

\section{Discussion}

BDNF has been described as a cancer related factor involved in breast cancer cell growth with a correlation to survival relevance in patients with breast cancer. However, the biological function of BDNF and the many cellular molecular pathways induced by BDNF are unknown (4).

The expression of BDNF and TrkB mRNA has been found to be higher in human cancer cell lines than in normal tissues (8). Our results also show mRNA expression level of BDNF in human breast cancer to be elevated and so we utilised RNA knockdown to study the influence of BDNF expression on cellular function and possible molecular mechanisms. In

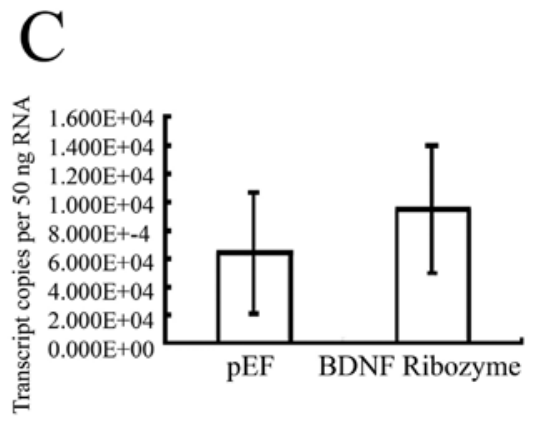

Figure 5. Effects of BDNF knockdown on the cellular signal pathway. (A) BDNF knockdown reduced the mRNA expression level of NFאB as shown by RT-PCR. (B) BDNF knockdown reduced the phosphorylation of AKT as shown using western blotting. (C) BDNF knockdown reduced the mRNA expression level of p53.

the present study we obtained stable knockdown of BDNF in human breast cancer cell lines using BDNF ribozymes.

When BDNF was stably knocked down in MDA-MB-231 and MCF-7 cell lines, the growth decreased compared with the vector control, suggesting that reduced BDNF gene expression could inhibit cellular proliferation. Cell proliferation was restored when stimulated by BDNF protein. BDNF is therefore involved in proliferation regulation of human breast cancer cell proliferation.

The apoptosis experiments demonstrated that in those MDA-MB-231 and MCF-7 cell lines in which BDNF was stably knocked down, apoptosis increased compared with the vector control. This suggests that BDNF is a regulator of apoptosis in these cells. It can be concluded that BDNF can maintain breast cancer cell survival and proliferation.

The influence of BDNF on cellular biological function is induced mainly by its receptor TrkB. When BDNF binds to TrkB the tyrosine kinase activity of the receptor is activated via phosphorylation of tyrosine residues in the cytoplasmic region of the receptor which in turn induces cellular signaling (13). The PI3K-AKT pathway is highly related to cell survival and PI3K has been shown to play a key role in anti-apoptotic survival and proliferation (14-16). We have found that phosphorylation of the AKT signal was weak in BDNF knocked down cells compared with vector control when treatment with BDNF. This result show that BDNF activates the AKT pathway in order to maintain cell survival (10). The MAPK pathway is known to be involved in proliferation but we did not find obvious changes in ERK1/2 phosphorylation in BDNF knocked down cells.

In this study, we also investigated the expression of downstream molecules related to the AKT pathway. The nuclear transcription factor, $\mathrm{NF} \kappa \mathrm{B}$ was found to be downregulation in the BDNF knocked down MDA-MB-231 cells but Bcl-2 demostrated no obvious changes (data not shown) compared with vector control. Therefore, the NFKB expression inhibition induced by BDNF downregulation may inhibit nucleolar transcription as a trigger for cell apoptosis (17). Accordingly, BDNF can facilitate NFKB expression to induce cell proliferation. 
We found that the mRNA transcription level of p53 was elevated in BDNF knocked down MDA-MB-231 cells compared with vector control. p53 is well-known to be a pro-apoptotic protein related to cell survival. p53 trancription increase induced by lower BDNF expression may increase cell apoptosis. BDNF has been found to mediate protection from apoptosis by $\mathrm{p} 53$ activation (18). High expression of BDNF and its receptor TrkB have been found in cancers related to metastasis and poor prognosis, in addition p53 is related in the late process of tumor progression and predict of a poor prognosis in squamous cell carcinoma of the uterine cervix $(4,19)$.

The BDNF downregulation induced abolishment of protection from apoptosis is affected by triggering downstream interplay between $\mathrm{NF \kappa B}$ activation and p53 inhibition. This abolishment is not effected by upregulation of the BDNFAkt-Bcl2 anti-apoptotic signaling pathway (15).

In conclusion, our study showed that BDNF facilitated cell proliferation and inhibited cell apoptosis in human breast cancer cells. Reduced BDNF expression induces changes in downstream signaling molecules, which are related to cell survival and apoptosis. BDNF is therefore a potential therapeutic target in breast cancer and its effect in human breast cancer requires further investigation.

\section{Acknowledgements}

We wish to thank Breast Cancer Hope Foundation and Cancer Research Wales for their support. Dr Yang is a recipient of the China Medical Scholarship of Cardiff University and sponsorship from the Albert Hung Foundation.

\section{References}

1. Foretova L, Petrakova K, Palacova M, Kalabova R, Svoboda M, Navratilova M, Schneiderova M, Bolcak K, Krejci E, Drazan L, et al: Genetic testing and prevention of hereditary cancer at the MMCI - over 10 years of experience. Klin Oncol 23: 388-400, 2010.

2. Gluck S and Mamounas T: Improving outcomes in early-stage breast cancer. Oncology 24: 1-15, 2011.

3. Barde YA, Edgar D and Thoenen H: Purification of a new neurotrophic factor from mammalian brain. EMBO J 1: 549-553, 1982

4. Patani N, Jiang WG and Mokbel K: Brain-derived neurotrophic factor expression predicts adverse pathological and clinical outcomes in human breast cancer. Cancer Cell Int 11: 23, 2011.

5. Vanhecke E, Adriaenssens E, Verbeke S, Meignan S, Germain E, Berteaux N, Nurcombe V, Le Bourhis X and Hondermarck H: Brain-derived neurotrophic factor and neurotrophin-4/5 are expressed in breast cancer and can be targeted to inhibit tumor cell survival. Clin Cancer Res 17: 1741-1752, 2011.
6. Yoshii A and Constantine-Paton M: Postsynaptic BDNF-TrkB signaling in synapse maturation, plasticity, and disease. Dev Neurobiol 70: 304-322, 2010.

7. Cowansage KK, LeDoux JE and Monfils MH: Brain-derived neurotrophic factor: a dynamic gatekeeper of neural plasticity. Curr Mol Pharmacol 3: 12-29, 2010.

8. Guo D, Hou X, Zhang H, Sun W, Zhu L, Liang J and Jiang X: More expressions of BDNF and TrkB in multiple hepatocellular carcinoma and anti-BDNF or K252a induced apoptosis, supressed invasion of HepG2 and HCCLM3 cells. J Exp Clin Cancer Res 30: 97, 2011.

9. Harada T, Yatabe Y, Takeshita M, Koga T, Yano T, Wang Y and Giaccone G: Role and relevance of TrkB mutations and expression in non-small cell lung cancer. Clin Cancer Res 17: 2638-2645, 2011.

10. Yu X, Liu L, Cai B, He Y and Wan X: Suppression of anoikis by the neurotrophic receptor TrkB in human ovarian cancer. Cancer Sci 99: 543-552, 2008

11. Festuccia C, Gravina GL, Millimaggi D, Muzi P, Speca S, Ricevuto $E$, Vicentini $C$ and Bologna M: Uncoupling of the epidermal growth factor receptor from downstream signal transduction molecules guides the acquired resistance to gefitinib in prostate cancer cells. Oncol Rep 18: 503-511, 2007.

12. Brunetto de Farias C, Rosemberg DB, Heinen TE, Koehler-SantosP, Abujamra AL, Kapczinski F, Brunetto AL, Ashton-Prolla P, Meurer L, Reis Bogo M, et al: BDNF/TrkB content and interaction with gastrin-releasing peptide receptor blockade in colorectal cancer. Oncology 79: 430-439, 2011.

13. Kawamura N, Kawamura K, Manabe $M$ and Tanaka T: Inhibition of brain-derived neurotrophic factor/tyrosine kinase B signaling suppresses choriocarcinoma cell growth. Endocrinology 151: 3006-3014, 2010

14. Li Z and Thiele CJ: Targeting Akt to increase the sensitivity of neuroblastoma to chemotherapy: lessons learned from the brainderived neurotrophic factor/TrkB signal transduction pathway. Expert Opin Ther Targets 11: 1611-1621, 2007.

15. Sheikh AM, Malik M, Wen G, Chauhan A, Chauhan V, Gong CX, Liu F, Brown WT and Li X: BDNF-Akt-Bcl2 antiapoptotic signaling pathway is compromised in the brain of autistic subjects. J Neurosci Res 88: 2641-2647, 2010.

16. Sun CY, Hu Y, Huang J, Chu ZB, Zhang L, She XM and Chen L: Brain-derived neurotrophic factor induces proliferation, migration, and VEGF secretion in human multiple myeloma cells via activation of MEK-ERK and PI3K/AKT signaling. Tumour Biol 31: 121-128, 2010.

17. D'Intino G, Vaccari F, Sivilia S, Scagliarini A, Gandini G, Giardino L and Calza L: A molecular study of hippocampus in dogs with convulsion during canine distemper virus encephalitis. Brain Res 1098: 186-195, 2006.

18. Kalita K, Makonchuk D, Gomes C, Zheng JJ and Hetman M: Inhibition of nucleolar transcription as a trigger for neuronal apoptosis. J Neurochem 105: 2286-2299, 2008.

19. Moon A, Won KY, Lee JY, Kang I, Lee SK and Lee J: Expression of BDNF, TrkB, and p53 in early-stage squamous cell carcinoma of the uterine cervix. Pathology 43: 453-458, 2011. 